\title{
Ecografía pulmonar y COVID-19
}

\section{Pulmonary ultrasound and COVID-19}

María Carolina Cabrera Schulmeyer ${ }^{1}$

\begin{abstract}
Pulmonary ultrasound has proven to be a tool that has been positioning itself in different specialties. Faced with the pandemic that affects us, this tool can be useful for making a diagnosis and screening of the state of the lung and thus generating a system that allows patients who have a normal or mildly compromised lung examination to be referred to their homes. While patients with greater lung involvement are admitted and managed in units of different complexity.
\end{abstract}

\section{RESUMEN}

El ultrasonido pulmonar ha demostrado ser una herramienta que ha ido posicionándose en diferentes especialidades. Frente a la pandemia que nos afecta esta herramienta puede ser útil para realizar un diagnóstico y un screening del estado del pulmón y así generar un sistema que permita derivar a sus casas a los pacientes que tengan un examen pulmonar normal o con leve compromiso. Mientras que los enfermos que tengan mayor compromiso pulmonar sean ingresados y manejados en unidades de diferente complejidad.

\section{Key words:}

pulmonary ultrasound, COVID-19

\section{Palabras clave:}

ultrasonido pulmonar, COVID-19

\section{Introducción}

El ultrasonido pulmonar puede ser una herramienta muy útil al momento de evaluar a un paciente portador de COVID-19.

Sólo se requiere conocer el examen pulmonar normal y los patrones característicos de síndrome alvéolo intersticial y el de consolidación pulmonar[1]-[3].
Tradicionalmente, el abordaje diagnóstico por la imagen del tórax en el paciente se ha basado en la radiografía simple anteroposterior. Sin embargo, ésta presenta grandes limitaciones en la precisión diagnóstica de la enfermedad pleuropulmonar. La introducción de la tomografía axial computarizada resolvió en gran medida este problema, pero con el doble inconveniente de las dosis de radiación y del inevitable

Médico Anestesióloga, Universidad de Valparaíso. Valparaíso, Chile.

Fecha de recepción: 18 de marzo de 2020

Fecha de aceptación: 20 de marzo de 2020

\section{ORCID}

https://orcid.org/0000-0002-5544-8000

Correspondencia:

María Carolina Cabrera Schulmeyer

Email: maria.cabrera@uv.cl 
traslado fuera de la unidad de hospitalización. En este contexto, la ecografía pulmonar, gracias a su portabilidad, se convierte en una técnica útil a la cabecera del paciente y fácilmente reproducible y sin radiaciones ionizantes y sin necesidad de trasladar al paciente dentro del hospital.

\section{Examen pulmonar normal}

En la ecografía pulmonar, las costillas, el aire del pulmón actúan como barreras para los ultrasonidos, y provocan artefactos que debemos reconocer e interpretar para un correcto diagnóstico. El ultrasonido pulmonar se ha ido desarrollando progresivamente tanto en sus aspectos conceptuales como de aplicación práctica a la cabecera de los pacientes[4]-[6].

El equipo de ecografía necesario para la realización de la técnica solo precisa de imagen bidimensional y en modo $\mathrm{M}$, pero actualmente casi todos los equipos modernos están dotados de otras posibilidades (doppler, color, armónicos, etc.). Más importante es la disponibilidad de sondas multifrecuencia o de varios tipos de sonda. Las sondas de frecuencias entre 3,5-5 MHz, utilizadas para la exploración abdominal y cardíaca, proporcionan una adecuada visualización de los planos profundos, y permiten la caracterización de las consolidaciones y el derrame pleural. Para las estructuras más superficiales, es decir, la pleura y los signos y los artefactos que se generan de ella, los transductores lineales de frecuencia superior a $5 \mathrm{MHz}$ proporcionan mucha mejor resolución. La forma de la sonda utilizada (lineal, convexa o sectorial) también dependerá de la zona a estudiar, y será convexa si el espacio intercostal es estrecho, para evitar la interferencia acústica que producen las costillas[7].

Los transductores sectoriales son adecuados para el examen del espacio pleural por vía intercostal directa, ya que presenta una visión muy estrecha en los campos cercanos y el espacio pleural se identifica por los artefactos.

En los pacientes la exploración se realiza en la posición de decúbito supino, que permite un fácil abordaje anterolateral (Figura 1). Son suficientes 4 áreas en cada hemitórax, y que lo dividen en 2 zonas, anterior y lateral, con la línea axilar anterior como divisoria. A su vez, cada zona se divide en una superior y otra inferior según una línea horizontal que atravesaría la unión del tercio medio con el inferior del esternón. La exploración comenzaría por la zona anterosuperior y acabaría por la lateroinferior, con cortes longitudinales y transversales. A veces es necesario el estudio de las zonas dorsales, para lo que es precisa una ligera inclinación del enfermo, que se logra con una leve aducción del brazo ipsolateral o sentándolo.

Al situar el transductor lineal en sentido longitudinal perpendicular a los espacios intercostales se visualizan cerca de los bordes laterales de la pantalla 2 interfases ecogénicas redondeadas con una marcada sombra acústica que corresponde a las costillas. Aproximadamente a medio centímetro por debajo del inicio de estas se sitúa una línea hiperecogénica horizontal que representa la pleura (Figura 2). Con transductores de alta frecuencia y gran resolución a poca profundidad se pueden observar las 2 capas, parietal y pleural, de unos $2 \mathrm{~mm}$ de anchura y de aspecto regular, separadas entre sí por el espacio pleural (0,3 mm). En conjunto, el borde superior de las sombras de las costillas y la línea pleural, componen una imagen que semeja el perfil de un murciélago, y por esto ha recibido la denominación.

Líneas A: líneas hiperecogénicas horizontales y paralelas que se sitúan a una distancia múltiplo de la que existe entre el transductor y la línea pleural (Figura 3).

Si aplicamos el modo $\mathrm{M}$, se distinguen 2 zonas bien diferenciadas, que configuran el signo de la orilla de playa (seashore sign): la parte superior, que corresponde a la pared torácica, formada por líneas horizontales paralelas (el mar), y la parte inferior, desde la pleura, de aspecto granulado, como arena de playa (Figura 4).

La principal característica que hay que observar es la presencia de deslizamiento o sliding pleural en la superficie de unión pleuropulmonar, que ocurre obligatoriamente con la insuflación y el vaciado pulmonar durante las fases respiratorias.

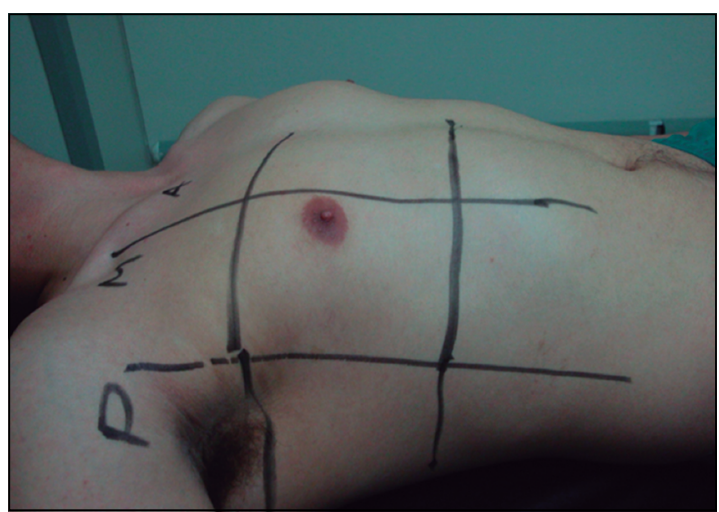

Figura 1. Esquema de cuadrantes ideal para subdividir el tórax. 


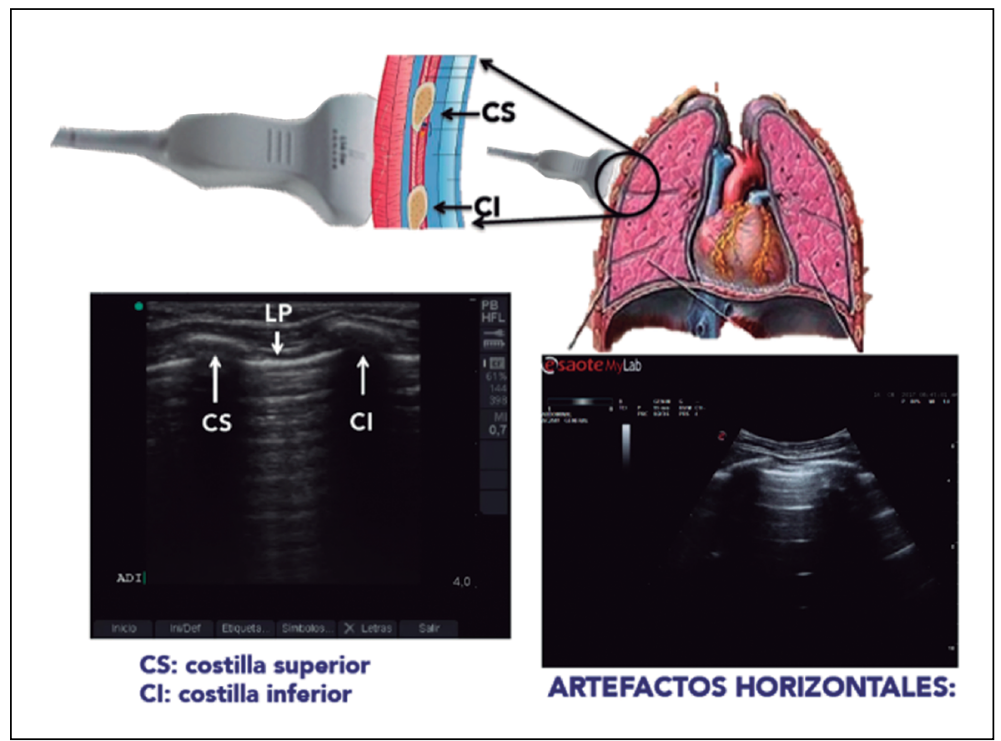

Figura 2. Examen pulmonar normal.

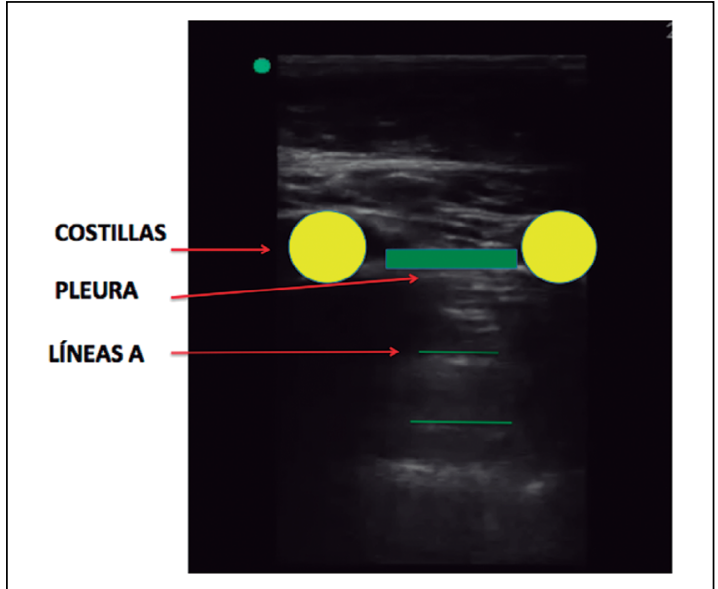

Figura 3. Examen pulmonar normal basado en artefactos.

\section{Síndrome alvéolo-intersticial}

La cantidad de líquido presente en el pulmón se verá reflejada con la aparición de líneas B (antiguas cola de cometa). Éstas son líneas hiperecoicas que van desde la pleura hasta la base de la pantalla y borran completamente las líneas A. Se forman cuando el haz de ultrasonido atraviesa dos interfases (aire y agua), generando un cambio en la impedancia acústica. Su número va en proporción directa a la gravedad del síndrome alvéolo intersticial que esté presente en el

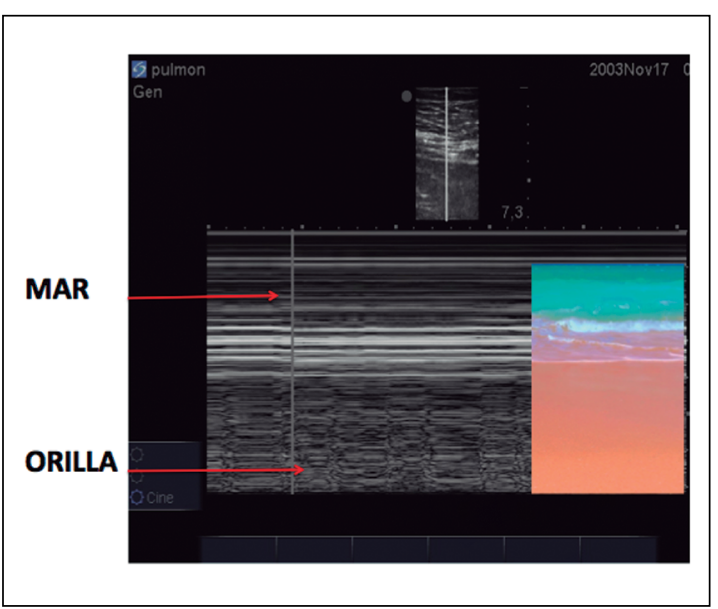

Figura 4. Patrón normal realizado con Modo M.

parénquima pulmonar y los síntomas que presentará este paciente. En el COVID-19 se ha visto la tendencia a un patrón parchado y distribuido en ambos campos pulmonares (Figura 6).

\section{Consolidación pulmonar}

Corresponde a la hepatización del parénquima pulmonar con la aparición de bronogramas aéreos y a la alteración completa del parénquima pulmonar. 


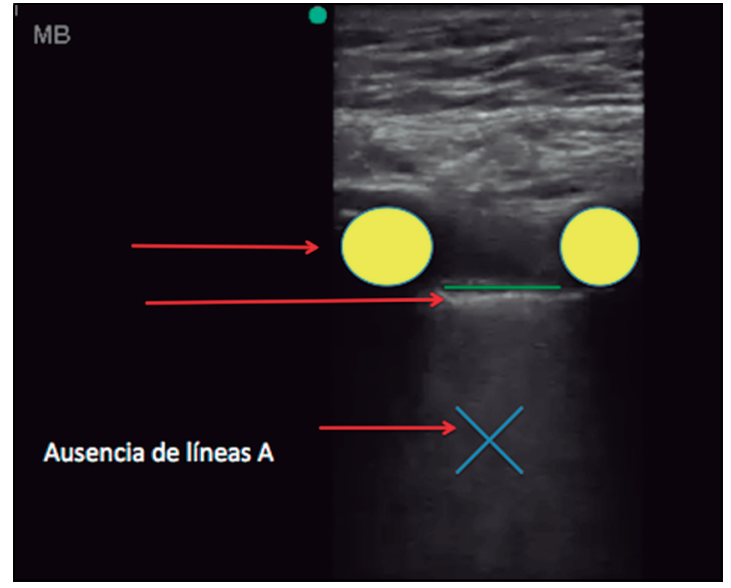

Figura 5. Patrón alvéolo intersticial con ausencia completa de líneas A que son reemplazadas por líneas $B$.

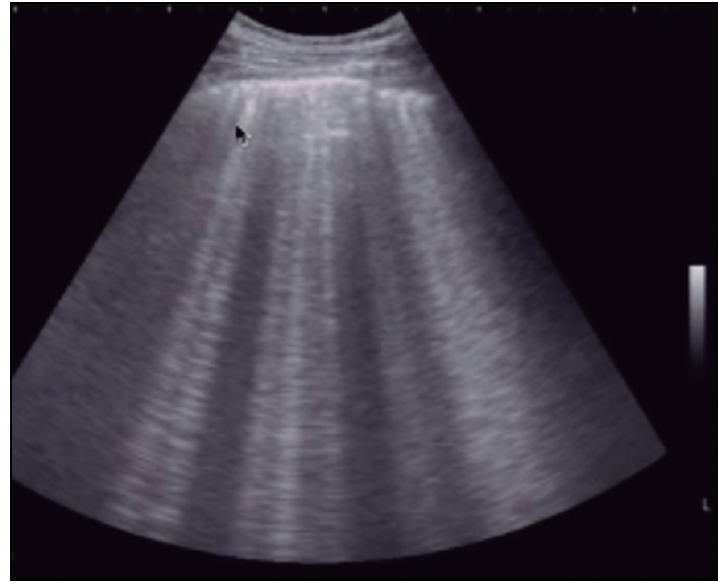

Figura 6. Pulmón con síndrome alvéolo intersticial con líneas B presentes.

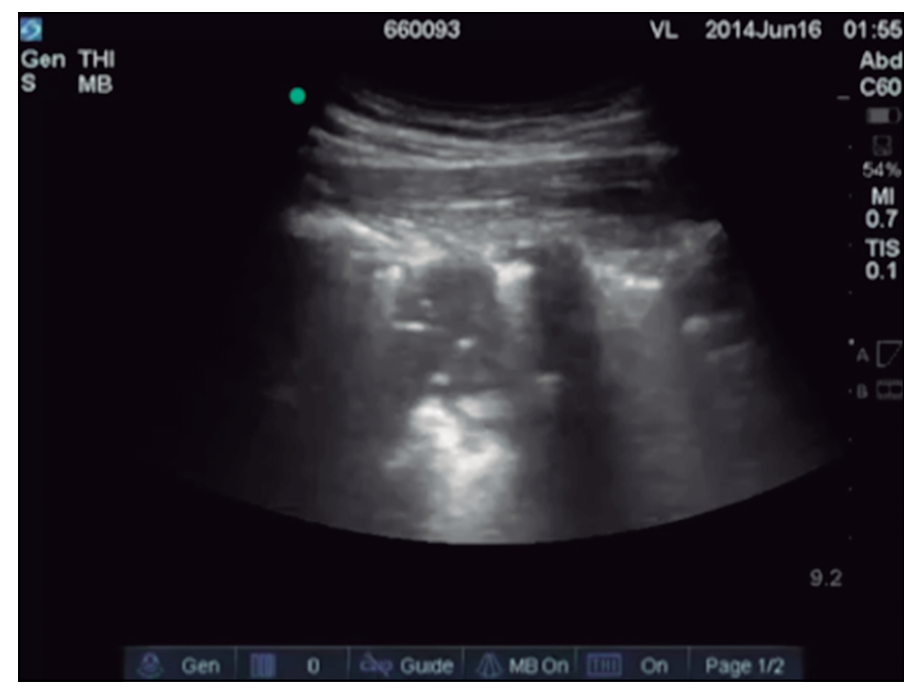

Figura 7. Consolidación pulmonar donde se observa el parénquima hepatizado y en su interior se presentan broncogramas aéreos.
Este patrón en pacientes portadores de COVID-19 es el que tiene un peor pronóstico con falla pulmonar catastrófica.

Este paciente debe ser ingresado a UCI y la última evidencia señala es que su intubación debiese ser lo más precoz posible (Figura 7).

\section{TRIAGE con US pulmonar}

El objetivo de éste es seleccionar los casos que pueden permanecer en sus domicilios y los que perentoriamente deben ser hospitalizados.
Para esto se utiliza una combinación entre el examen clínico, la saturometría del paciente y su US pulmonar.

1. Si el paciente presenta COVID (+) tiene saturometría normal y su US pulmonar tiene líneas A, debiese realizar cuarentena en casa con medidas generales.

2. Si el paciente presenta COVID (+) tiene saturometría normal y su US pulmonar tiene líneas B, puede realizar cuarentena escasa bajo estricto control y ojalá control telefónico del personal del hospital. 


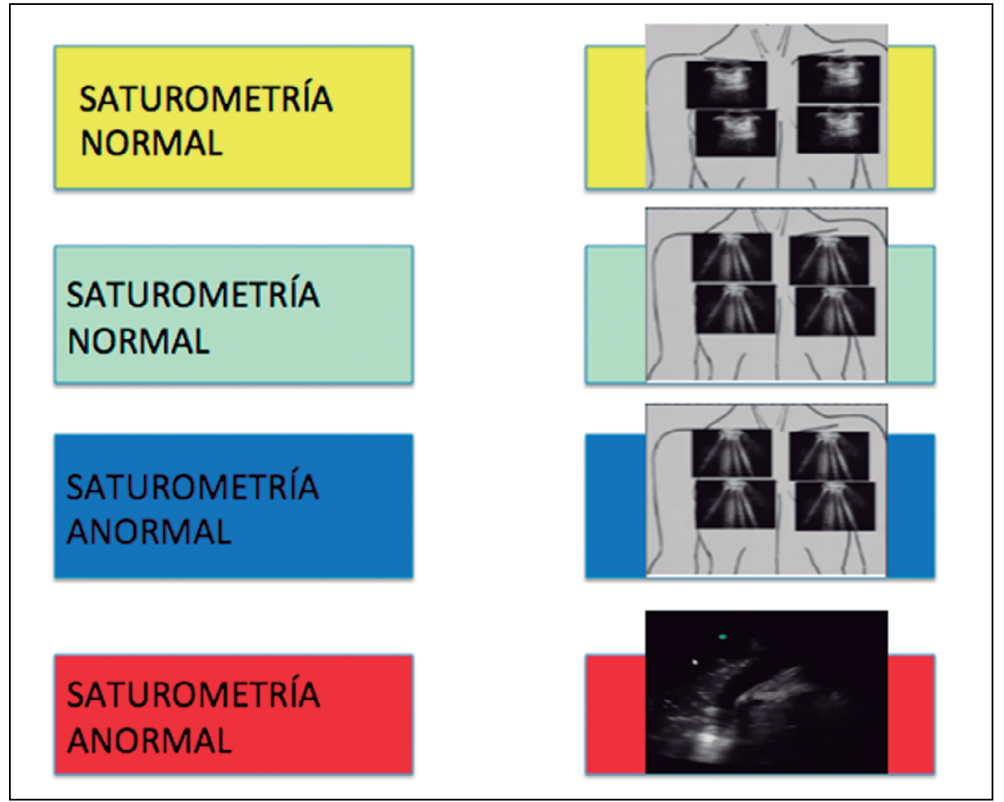

Figura 8. Esquema propuesto para manejo de pacientes COVID-19 (+) y US pulmonar.
3. Si el paciente presenta COVID (+) su saturometría es anormal y tiene líneas B debe ser hospitalizado ojalá en una Unidad de Pacientes Críticos y evaluar su evolución. Puede que no necesite ventilación mecánica.
4. Si el paciente presenta COVID (+) su saturometría es anormal y tiene patrón de consolidación, debe ser hospitalizado en una Unidad de Pacientes Críticos con la idea de instalarle ventilación mecánica (Figura 8).

\section{Referencias}

1. Lichtenstein DA. Lung ultrasound in the critically ill. Ann Intensive Care. 2014 Jan;4(1):1-8. https://doi. org/10.1186/2110-5820-4-1 PMID:24401163

2. Xirouchaki $N$, Kondili E, Prinianakis G, Malliotakis P, Georgopoulos D. Impact of lung ultrasound on clinical decision making in critically ill patients. Intensive Care Med. 2014 Jan;40(1):57-65. https://doi. org/10.1007/s00134-013-31333 PMID:24158410

3. Volpicelli $G$, Elbarbary $M$, Blaivas M, Lichtenstein DA, Mathis G, Kirkpatrick AW, et al.; Inter- national Liaison Committee on Lung Ultrasound (ILC-LUS) for International Consensus Conference on Lung Ultrasound (ICC-LUS). International evidence-based recommendations for point-of-care lung ultrasound. Intensive Care Med. 2012 Apr;38(4):577-91. https:// doi.org/10.1007/s00134-0122513-4 PMID:22392031

4. Gargani L, Volpicelli G. How I do it: lung ultrasound. Cardiovasc Ultrasound. 2014 Jul;12(1):25-30. https://doi. org/10.1186/1476-7120-12-25 PMID:24993976

5. Wongwaisayawan S, Suwannanon R, Sawatmongkorngul S, Kaewlai
R. Emergency Thoracic US: the essentials. Radiographics. 2016 May-Jun;36(3):640-59. https:// doi.org/10.1148/rg.2016150064 PMID:27035835

6. Lichtenstein DA. BLUE-protocol and FALLS-protocol: two applications of lung ultrasound in the critically ill. Chest. 2015 Jun;147(6):1659-70. https:// doi.org/10.1378/chest.14-1313 PMID:26033127

7. Lichtenstein DA, Lascols N, Mezière G, Gepner A. Ultrasound diagnosis of alveolar consolidation in the critically ill. Intensive Care Med. 2004 Feb;30(2):276-81. https://doi. org/10.1007/s00134-003-20756 PMID:14722643 